\section{EDUCATION}

Research, Innovation and Solutions on-line ${ }^{(0)}$

\section{Electronic Journal of Research}

in Educational Psychology

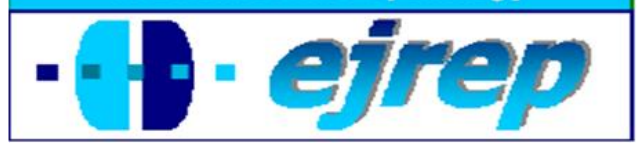

\title{
El impacto negativo del contrato didáctico en la resolución realista de problemas. Un estudio con alumnos de $2^{\circ}$ y $3^{\circ}$ de Educación Primaria
}

\author{
Jiménez, Laura y Ramos, Francisco J.
}

Facultad de Educación, Universidad de Castilla-La Mancha, Cuenca

\section{España}

Correspondencia: Laura Jiménez Márquez. Departamento de Psicología. Facultad de Educación. Campus Universitario s/n. 16071. Cuenca. España. E-mail: laura.jimenez@uclm.es

(C) Education \& Psychology I+D+i and Editorial EOS (Spain) 


\section{Resumen}

Introducción. Este estudio tuvo como objetivo analizar el impacto de algunas de las creencias incorrectas que los niños tienen acerca de las matemáticas sobre el fracaso en la resolución de problemas no rutinarios de adición.

Método. Se presentaron individualmente a alumnos de $2^{\circ}$ y $3^{\circ}$ de Primaria cuatro tipos de problemas no rutinarios, contrarios a dichas creencias. En concreto, los alumnos debían resolver problemas que omiten información relevante para hallar una solución, problemas con soluciones múltiples, problemas cuya solución no requiere cálculo y problemas que incluyen datos irrelevantes.

Resultados. Los resultados del ANOVA pusieron de manifiesto, en primer lugar, que el porcentaje de respuestas realistas fue muy bajo (en torno al 33\%) y, en segundo lugar, que los problemas que omiten información relevante y tienen soluciones múltiples resultan más difíciles para los alumnos que aquellos cuya solución no requiere cálculo y los que incluyen datos irrelevantes.

Discusión. Efectivamente, las creencias incorrectas de los niños sobre las matemáticas parecen ser las responsables del fracaso de los niños en la resolución de problemas no rutinarios. Los procedimientos de resolución y las soluciones ofrecidas por lo niños mostraron que no se percibe la relación entre las matemáticas escolares y las matemáticas del mundo real.

Palabras Clave: problemas no rutinarios, contrato didáctico, creencias incorrectas, educación matemática 


\title{
The negative impact of the didactic contract in realistic problem solving: a study with second- and third-grade students
}

\begin{abstract}
Introduction. This study aimed to analyze the impact of some children's incorrect beliefs about mathematics on the students' failure in non-routine adition problem solving.

Method. 22 second-graders and 22 third-graders were individually asked to solve four types of non-routine problems, against to those children's beliefs. Specifically, students had to solve problems omitting relevant information to find the solution, problems with multiple solutions, problems which solution requires no calculation and problems including irrelevant data.
\end{abstract}

Results.The ANOVA results showed, firstly, that the percentage of the realistic responses was very low (around 33\%) and, secondly, that the problems omitting relevant information and having multiple solutions were more difficult for students than problem requiring no calculations or including irrelevant data.

Discussion. Indeed, children's incorrect beliefs about mathematics seem to be responsible for children's failure in non-routine problem solving. Children's resolution procedures and solutions showed a gap between school and real life mathematics.

Keywords: Non-routine problems, didactical contract, incorrect beliefs, mathematics education 


\section{Introducción}

Uno de los principios básicos que ha sido defendido por múltiples investigadores en los últimos años es que la enseñanza de las matemáticas debe estar basada en la comprensión y solución de problemas verbales, ya que éstos constituyen contextos significativos para los niños (p.e., Becker y Selter, 1996; Burkhardt, 1994; Ellerton y Clarkson, 1996; Niss, 1996; Vicente, Van Dooren, y Verschaffel, 2008). Adicionalmente, se ha propuesto que los problemas escolares deben cumplir dos características principales: por un lado, deben constituir auténticos problemas, en contraposición a la descripción de situaciones que representen fielmente un procedimiento algorítmico de resolución, sin que sea necesario un proceso profundo de reflexión previo y, por otro, deben describir situaciones cercanas a la vida real de los niños y no meros enunciados carentes de sentido.

Para ello, muchos investigadores abogan por la introducción, en las tareas escolares, de problemas no rutinarios, es decir, auténticos para los alumnos. Así, muchos de los problemas rutinarios, típicamente escolares, pueden resolverse empleando procesos simples de pensamiento (p.e., "Un tendero tiene dos cajas de manzanas. La primera contiene 60 manzanas y la otra 90 manzanas. Si pone todas las manzanas juntas dentro de una caja más grande, ¿cuántas manzanas habrá en la nueva caja?” sería un problema típico escolar que no supone demasiada dificultad para los niños), mientras que los problemas no rutinarios serían aquéllos implican la puesta en marcha de procesos de reflexión más complejos (p.e., en el problema “¿Cuál será la temperatura de agua de un recipiente si ponemos un litro de agua a $80^{\circ}$ y un litro de agua a $40^{\circ}$ dentro de él?", la respuesta final debe resultar del cálculo de la temperatura media y no de la adición de las temperaturas de ambos recipientes) (ver p.e., Caldwell, 1995; Hidalgo, 1997; Reusser y Stebler, 1997; Verschaffel, de Corte y Lasure, 1994; Verschaffel, de Corte y Lasure, 1999; Yoshida, 1997).

Unido a esto, los problemas no rutinarios deberían formularse de forma que tengan sentido en la vida real. Esto es, en palabras de Verschafel, Greer y De Corte (2000), una de las ventajas de los problemas escolares es que podrían ser un posible nexo entre las matemáticas puras y su aplicación para resolver problemas reales en la vida. Desde este punto de vista, se considera que el aprendizaje de las matemáticas proviene de la necesidad de dar sentido a las situaciones a las que nos enfrentamos, hecho que debería ser respetado en clase favoreciendo 
que se establezcan conexiones entre la verosimilitud de los problemas que se presentan y las experiencias vividas por los niños (Verschaffel, Greer, Van Dooren y Mukhopadhyay, 2009). Estas tareas, más realistas para los niños, les permitirían emplear de forma crítica los procedimientos matemáticos en contextos significativos ajenos al puramente escolar (Blum y Niss, 1991; Villegas, Castro y Gutierrez, 2009).

Desde nuestro punto de vista, ambas características, esto es, la autenticidad de los problemas y el realismo, deberían estar presentes en las tareas matemáticas escolares.

\section{Las investigaciones con problemas no rutinarios}

Una gran cantidad de estudios, realizados desde 1980 hasta la actualidad, han demostrado que los niños muestran una fuerte tendencia a no usar el conocimiento del mundo real cuando resuelven problemas, no considerando, por tanto, que su respuesta debería tener sentido en situaciones semejantes a las que suceden fuera de la escuela. Igualmente, en estas investigaciones se asume que una de las principales causas es que los niños no asumen como equivalentes los problemas escolares y los problemas de la vida real.

Así, Baruk (1980) demostró que los niños, en un contexto escolar, son capaces de resolver cualquier afirmación que sea seguida de un interrogante, con independencia de lo absurdo que suponga hacer esto. Para ello, presentó a alumnos de $1^{\circ}$ a $4^{\circ}$ de Educación Primaria (E.P.) el siguiente problema: "Hay 26 ovejas y 10 cabras en un barco. ¿Cuántos años tiene el capitán?”. Sorprendentemente, el $88 \%$ de los niños de $1^{\circ}$ y $2^{\circ}$ de E.P. y el $38 \%$ de los niños de $3^{\mathrm{o}}$ y $4^{\mathrm{o}}$ resolvieron el problema sumando las ovejas y las cabras para conocer la edad del capitán.

Otros muchos autores se han planteado investigar los procedimientos de resolución de los niños formulando problemas no rutinarios basados en el mundo real, es decir, problemas que son cercanos a la vida real de los niños, pero que no pueden resolverse matemáticamente mediante la operación aritmética más obvia. Así, Greer (1993) y Verschaffel et al. (1994) encontraron que tan sólo el $17 \%$ de las soluciones de los niños, como media, podían considerarse respuestas apropiadas a los problemas no rutinarios presentados (ver también Caldwell, 1995; Hidalgo, 1997; Reusser y Stebler, 1997; Verschaffel et al., 1999; Yoshida, 1997). Por ejemplo, en el problema del "Agua”, enunciado anteriormente, tan sólo el 17\% de las respues- 
tas de los niños eran correctas (i.e., $60^{\circ}$ ), puesto que muchos de ellos optaban por adicionar la temperatura de ambos recipientes (i.e., 120\%). De forma adicional, Caldwell (1995), en un estudio de réplica, se planteó ahondar en los motivos que llevaban a los niños a resolver los problemas de acuerdo a procedimientos estereotipados de resolución, y encontró que algunos de los alumnos que erraban justificaban la elección de un procedimiento equivocado aludiendo a las diferencias que presumiblemente existen entre los problemas del mundo real y los problemas matemáticos (p.e., “Conozco esas cosas, pero yo nunca pensaría incluirlas en un problema de matemáticas. Las matemáticas tratan acerca de cómo hacer sumas bien y esas cosas no son necesarias para hacer bien las sumas") (Caldwell, 1995, p. 39). En resumen, el resultado de las entrevistas sugirió que los niños tienden a resolver los problemas de forma estereotipada debido a que no consideran útil las demandas que los problemas plantean.

Otros ejemplos de la falta de realismo en las respuestas de los niños han sido igualmente obtenidos con problemas de división con resto como, por ejemplo, el famoso problema del "Autobús" (i.e., "Un autobús del ejército tiene 36 plazas. Si van a trasladar 1128 soldados ¿cuántos autobuses se necesitan?”), empleado en la Tercera Evaluación Nacional sobre el Progreso Educativo (NAEP, 1983) y en el Test de Matemáticas del Programa de Evaluación de California (CAP, 1983). Los resultados de ambos estudios mostraron que aproximadamente el $19 \%$ de ellos pensaban que se necesitarían 31 autobuses, dejando "en tierra" a 12 soldados, mientras que el $29 \%$ de los niños consideraban que serían necesarios 31.33 autobuses (para una revisión de los estudios dedicados a los problemas de división con resto ver también Cai y Silver, 1995; Carpenter, Linkquist, Matthews y Silver, 1983; Lago, Rodríguez, Enesco, Jiménez y Dopico, 2008; Li y Silver, 2000; Rodríguez, Lago, Hernández, Jiménez, Guerrero y Caballero, 2009; Silver, 1986; Silver, Mukhopadhyay y Gabriele, 1992; Silver, Shapiro y Deutsch, 1993).

\section{¿Qué características debemos considerar a la hora de formular problemas no rutinarios?}

Es cierto que, a la hora emular todos los aspectos que suceden en la vida real en los problemas matemáticos, existen ciertas limitaciones. Por ese motivo, algunos de los problemas que se han empleado en estudios previos podrían considerarse cuasi-auténticos o pseudorealistas, debido a requieren de los estudiantes la formulación de preguntas que no son realmente habituales en la vida real (Boaler, 1993; Nesher, 1980; Jiménez, 2008; Verschaffel et al., 2000). 
De acuerdo con Palm (2006, 2008, 2009), a la hora de juzgar si un problema es auténtico, hay que tener en cuenta una serie de descriptores. El primero de ellos sería la propia situación descrita, ya que es importante que sean eventos ocurridos en la vida de los niños, que sea posible que sucedan o que sean conocidos. Por ejemplo, en el problema del "Agua", mencionado anteriormente, la situación descrita no es muy habitual para los niños e, incluso, podrían tener conocimientos erróneos sobre ella. De hecho, este mismo problema se administró, durante el transcurso de una clase ordinaria, a estudiantes universitarios de $1^{\circ}$ de Grado en Maestro de Educación Primaria. Aproximadamente, el 10\% de los futuros profesores consideraba que la solución era $40^{\circ}$ puesto que "la temperatura más fría debe substraerse a la más caliente" e, igualmente, el $5 \%$ que la respuesta al problema eran $120^{\circ}$ porque "ambos recipientes contienen agua caliente" (resultados obtenidos en pruebas piloto realizadas en varios grupos durante los cursos académicos 2009-10 y 2010-11).

El segundo elemento de análisis es la pregunta final a la que deben dar respuesta. Ésta ha de indicar claramente cuál es el problema a resolver y debe reflejar cuestiones que uno podría llegar a plantearse tras leer el enunciado. En el problema anterior, conocer la temperatura final tras mezclar dos litros de agua caliente con distintas temperaturas.

El tercer descriptor es la representatividad de la información o los datos incluidos. En este sentido, no parece muy probable que los niños se hayan enfrentado a situaciones en las que hayan tenido que calcular el resultado de mezclar temperaturas de $40^{\circ}$ y $80^{\circ}$. Quizás, hubiese podido ser más cercana para ellos la mezcla de dos recipientes, uno con agua a $30^{\circ} \mathrm{y}$ otro a $50^{\circ}$, para llenar una bañera.

En cuarto lugar, es igualmente importante no olvidar que existen múltiples estrategias para solucionar un problema y no sólo los algoritmos. En la vida real, los niños suelen emplear estrategias que ellos mismos han desarrollado. Además, tampoco podemos presuponer que exista una única respuesta realista. Por ejemplo, en el problema de los "Amigos", formulado originalmente por Nelissen (1987), y empleado en el estudio de Verschaffel et al. (1994) (i.e. "Carlos tiene 5 amigos y Jorge tiene 6 amigos. Carlos y Jorge deciden celebrar juntos su cumpleaños e invitan a todos sus amigos. Todos sus amigos asisten a la fiesta. ¿Cuántos amigos asisten?”), la dificultad proviene precisamente de que la solución correcta puede ser cual- 
quier número comprendido entre 6 y 11, sin contar a Carlos y Jorge, puesto que pueden tener hasta 5 amigos comunes.

La forma de presentación del problema debe ser igualmente tenida en cuenta. En la vida real los problemas se transmiten mediante comunicación oral, mientras que en la escuela se presentan en un formato escrito $y$, a veces, se acompañan de gráficos o tablas que son difíciles de interpretar. En este sentido, sería importante que los problemas pudieran ser transmitidos de formas diversas como, por ejemplo, contados o leídos por el profesor u otros compañeros.

Por último, las circunstancias en las que los niños suelen resolver los problemas también son diferentes. En la vida real es posible acudir a otra persona, discutir con ella acerca de cómo solucionar el problema o pedirle ayuda, pero en las tareas escolares se suelen resolver los problemas de forma individual y, además, en un tiempo predeterminado, lo que afectaría negativamente al nivel de éxito. Buena prueba de ello es el estudio de Verschaffel et al. (1999) en el que se plantearon evaluar a niños de $5^{\circ}$ de E.P., empleando la versión original del problema de los "Amigos" y una segunda versión en la que se introducía un andamiaje (i.e., “¿Puedes decirme el nombre de un buen amigo de clase? Imagina que tú y ese amigo dais una fiesta juntos y que ambos invitáis a vuestros mejores amigos. Imagina que tus 5 mejores amigos van y los 6 mejores amigos de ese amigo van también. ¿Estás seguro de que habrá 11 invitados en la fiesta?”). Así, mientras que en la versión inicial, presentada como un problema típicamente escolar, el índice de aciertos se situó en el 23\%, en la segunda presentación el $57 \%$ de los niños identificaron que había múltiples respuestas posibles, lo que muestra que, efectivamente, las circunstancias en las que se presentan los problemas influyen en el grado de realismo que los niños consideran aceptable considerar en sus soluciones.

\section{Ahondando en el origen de las dificultades de los niños}

Múltiples investigaciones han hecho referencia al impacto negativo que tiene la aplicación masiva de problemas rutinarios en la forma en la que los alumnos consideran que deben resolver dichos problemas. Así, los niños consideran que deben seleccionar sistemáticamente todas las cantidades y aplicar la operación aritmética más obvia, basándose en la detección de una palabra clave en el texto (ver p.e., Carpenter, Hiebert y Moser, 1983; Fuson, 1992; Hegarty, Mayer y Monk, 1995; Orrantia, 2003; Orrantia, González y Vicente, 2005; 
Verschaffel y de Corte, 1997). De hecho, este tipo de proceder se perpetúa debido a que, en la mayor parte de los casos, conduce a los alumnos al éxito. Estas normas y creencias implícitas acerca de cómo deben resolverse los problemas matemáticos están inmersas en lo que se conoce con el nombre de contrato didáctico (p.e., Brousseau, 1984; Greer, 1993; Reusser, 1988; Reusser y Stebler, 1997; Schoenfeld, 1991; Verschaffel et al., 2000; Wyndhamn y Säljö, 1997). Aunque los problemas cumplan todos los requisitos de autenticidad descritos anteriormente, es importante que los estudiantes se sientan libres de resolver los problemas como si estuvieran en el mundo real, en contraste con lo que consideran que se espera de ellos en un contexto escolar.

Desde este punto de vista, se propone que el fracaso de los alumnos estaría motivado por estas creencias, compartidas igualmente por los profesores, y que se encuentran en la base del contrato didáctico (p.e., Caldwell, 1995; Gil, Guerrero y Blanco, 2006; Greer, 1993; Hidalgo, 1997; Verschaffel et al., 1994, 1999). Siguiendo a Reusser y Stebler (1997), algunas de las creencias incorrectas más importantes serían: (1) todo problema tiene una solución; (2) hay una única solución, numérica y precisa, para cada problema; (3) la solución se obtiene aplicando una operación aritmética y (4) todos los números que figuran en el enunciado deben ser empleados en los cálculos.

\section{Objetivos de la investigación}

Precisamente, el propósito de esta investigación es profundizar en el impacto que tienen estas cuatro asunciones del contrato didáctico en la resolución de problemas no rutinarios. Para ello, se han diseñado cuatro tipos de problemas que, aunque describen situaciones cercanas para los niños, tienen la dificultad de que violan dichas creencias erróneas. En concreto, se han formulado problemas que Omiten Información Relevante (en contra de la creencia de que todos los problemas son resolubles), con Soluciones Múltiples (que atentan a la convicción de que hay una única solución numérica precisa), cuya Solución no requiere Cálculo (contrarios a la consideración de que siempre se ha de aplicar una operación aritmética para resolver un problema) y que incluyen Datos Irrelevantes (opuestos a la idea de que todos los datos numéricos deben utilizarse en los cálculos).

De forma adicional, y dado que la mayoría de los estudios previos han empleado grupos de alumnos provenientes de un mismo nivel escolar, el segundo objetivo de este estudio 
ha sido analizar posibles diferencias existentes en los tipos de respuesta, correctas e incorrectas, ofrecidas por niños de dos niveles escolares diferentes, $2^{\circ}$ y $3^{\circ}$ de Educación Primaria.

\section{Método}

\section{Participantes}

Participaron un total de 44 alumnos procedentes de un colegio público: 22 alumnos de $2^{\circ}$ de E.P. ( $(M=7 ; 7$ años $)$ y 22 alumnos de $3^{\circ}$ de E.P. $(M=8 ; 6$ años $)$.

\section{Material y Procedimiento}

Todos los alumnos fueron evaluados, mediante una entrevista individual, empleando una tarea de resolución de problemas verbales. En concreto, se formularon cuatro Tipos de problema en función de la Información ofrecida en el Enunciado, esto es, problemas que Omiten Información Relevante, con Soluciones Múltiples, cuya Solución no requiere Cálculo y que incluyen Datos Irrelevantes. Cada participante resolvió dos ensayos de cada uno de ellos (ver Tabla 1).

Con respecto a la elaboración del material, se confeccionaron dos cuadernillos, uno para cada ensayo, en los que se incluían, además de los cuatro problemas no rutinarios señalados anteriormente, dos problemas rutinarios adicionales de resta que actuaban como distractores. El orden de los problemas en cada cuadernillo se determinó al azar y se mantuvo constante durante toda la aplicación. Además, para evitar que los alumnos pudiesen advertir que en ambas ocasiones, esto es, en el ensayo 1 y en el ensayo 2, se describían situaciones similares, entre ambas aplicaciones se dejó transcurrir una semana. Por tanto, todos los participantes resolvieron un total de 8 problemas no rutinarios y 4 problemas distractores.

Adicionalmente, para evitar posibles dificultades derivadas de otros factores que han probado afectar a la ejecución de los niños (i.e., la estructura semántica, la ubicación de la incógnita y el tamaño de las cantidades, entre otros), todos los problemas se formularon con una estructura semántica sencilla de cambio, la incógnita se ubicó en el resultado y las cantidades oscilaron, en el primer sumando, entre 10 y 19 y, en el segundo, entre 5 y 9 . Asimismo, a fin de evitar posibles dificultades relacionadas con la comprensión lectora, todos los pro- 
blemas, además de ser presentados por escrito en los cuadernillos correspondientes, eran leídos previamente en voz alta por el investigador y dicha lectura se repetía tantas veces como el niño lo requiriese.

Tabla 1. Tipos de problema en función de la Información ofrecida en el Enunciado

\begin{tabular}{|c|c|c|}
\hline & Ensayo 1 & Ensayo 2 \\
\hline & Problema del "Circo" & Problema de las "Canicas" \\
\hline $\begin{array}{l}\text { Omiten In- } \\
\text { formación } \\
\text { Relevante }\end{array}$ & $\begin{array}{l}\text { María ha ido al circo con sus ami- } \\
\text { gas. María tiene } 13 \text { euros y su ami- } \\
\text { ga Alicia le deja } 7 \text { euros para pagar } \\
\text { la entrada. ¿Cuánto dinero le que- } \\
\text { da a Alicia? }\end{array}$ & $\begin{array}{l}\text { Mario ha bajado al parque a jugar a } \\
\text { las canicas con sus amigos. Mario } \\
\text { tiene } 17 \text { canicas y su amigo Jorge le } \\
\text { da } 7 \text {. ¿Cuántas canicas le quedan a } \\
\text { Jorge? }\end{array}$ \\
\hline & Problema de los "Chicles" & Problema de los “Pasteles" \\
\hline Soluciones & $\begin{array}{l}\text { Ana ha comprado una bolsa de } 14 \\
\text { chicles de varios sabores. Como sus } \\
\text { preferidos son los de menta y le }\end{array}$ & $\begin{array}{l}\text { Lorena ha comprado } 16 \text { pasteles } \\
\text { de varios sabores en la pastelería. } \\
\text { Como ve que le han puesto pocos }\end{array}$ \\
\hline Múltiples & $\begin{array}{l}\text { han puesto pocos, Ana compra } \\
\text { también } 8 \text { chicles de menta. } \\
\text { ¿Cuántos chicles de menta tiene } \\
\text { ahora Ana? }\end{array}$ & $\begin{array}{l}\text { de nata y son sus preferidos, Lore- } \\
\text { na pide también } 7 \text { pasteles de na- } \\
\text { ta. ¿Cuántos pasteles de nata tiene } \\
\text { ahora Lorena? }\end{array}$ \\
\hline $\begin{array}{l}\text { La Solución } \\
\text { no requiere } \\
\text { Cálculo }\end{array}$ & $\begin{array}{l}\text { Problema de la "Granja" } \\
\text { Un pastor tiene } 17 \text { ovejas en su } \\
\text { granja. Como quiere ampliar la } \\
\text { granja compra } 8 \text { cabras. ¿Cuántas } \\
\text { ovejas tiene ahora el pastor en la } \\
\text { granja? }\end{array}$ & $\begin{array}{l}\text { Problema del "Zoo" } \\
\text { En el zoo de Madrid tienen } 16 \text { leo- } \\
\text { nes. Como tienen mucho terreno } \\
\text { libre compran } 6 \text { tigres. ¿Cuántos } \\
\text { leones hay ahora en el zoo? }\end{array}$ \\
\hline & Problema de las “Pinturas" & Problema de las "Acuarelas" \\
\hline $\begin{array}{l}\text { Datos Irre- } \\
\text { levantes }\end{array}$ & $\begin{array}{l}\text { Laura ha comprado una caja de } 12 \\
\text { pinturas para clase de Plástica. Su } \\
\text { amiga Silvia le regala otra caja que } \\
\text { contiene } 3 \text { bolígrafos y } 9 \text { pinturas. } \\
\text { ¿Cuántas pinturas tiene ahora Lau- } \\
\text { ra? }\end{array}$ & $\begin{array}{l}\text { Lourdes tiene una caja de } 14 \text { acua- } \\
\text { relas de colores. Su amiga Mónica } \\
\text { le regala otra caja que contiene } 4 \\
\text { témperas y } 7 \text { acuarelas de colores. } \\
\text { ¿Cuántas acuarelas de colores tie- } \\
\text { ne ahora Lourdes? }\end{array}$ \\
\hline
\end{tabular}

Análisis y Codificación de las Respuestas

La categorización de las respuestas se llevó a cabo mediante un acuerdo entre tres jueces. Para ello, se tuvieron en cuenta conjuntamente la operación aritmética empleada y las justificaciones que ofrecieron para fundamentar su respuesta. Finalmente, todas las respuestas se dividieron en dos grandes grupos: 


\section{Respuestas Realistas}

- Respuestas Realistas Correctas. En esta categoría se recogieron todas las respuestas que fueron correctas, tanto por la elección correcta del algoritmo, en el caso que fuera necesario operar (i.e., en los problemas con Soluciones Múltiples y Datos Irrelevantes), como por la justificación de la respuesta final. Fueron las únicas respuestas que se consideraron correctas, asignándoles una puntuación cuantitativa de un punto.

- Respuestas Realistas Incorrectas. Esta categoría comprendía los comentarios realistas asociados a una respuesta numérica incorrecta, debido a que los alumnos consideraron parcialmente aspectos realistas de la situación, pero finalmente optaron por soluciones matemáticas que se ajustaban a sus creencias.

\section{Respuestas No Realistas}

- Respuestas Esperadas. Dado que todos los problemas tenían una estructura semántica aditiva de cambio y la incógnita se situaba en el resultado, esta respuesta siempre consistió en la suma de todas las cantidades numéricas, esto es, los dos datos en el caso de los problemas que Omiten Información Relevante, con Soluciones Múltiples y cuya Solución no requiere Cálculo y los tres dígitos en los que incluían Datos Irrelevantes.

- Otras Respuestas Incorrectas. En esta categoría se consignaron las soluciones que estaban basadas en procedimientos algorítmicos ajenos a la adición (i.e., sustracción, multiplicación, división y distintas combinaciones de las cuatro operaciones aritméticas básicas). En todos los casos, el procedimiento de resolución llevaba aparejadas justificaciones basadas en la lectura superficial del texto o la alusión a palabras "clave".

- Ausencia de Respuesta. En este caso, el alumno no fue capaz de elaborar ninguna solución numérica, ni de explicar de dónde procedía su dificultad para resolver el problema.

\section{Análisis de datos}

Para el análisis cuantitativo de los datos, se consideraron correctas y, por tanto, se les asignó un punto a todas las Respuestas Realistas Correctas y cero puntos al resto de solucio- 
nes (i.e., Respuestas Realistas Incorrectas, Respuestas Esperadas, Otras Respuestas Incorrectas y Ausencia de Respuesta). Por tanto, en cada Tipo de Problema según la Información Ofrecida en el Enunciado, la puntuación final podía ser 0 puntos si no se había contestado correctamente a ninguno de los dos ensayos, 1 punto en el caso de que se hubiera ofrecido una Respuesta Realista en uno de ellos y 2 puntos si ambos ensayos se habían resuelto correctamente. Para el análisis estadístico de los datos se aplicó un ANOVA mixto.

\section{Resultados}

El ANOVA mixto 2 (Grupo: $2^{\circ}$ vs. $3^{\circ}$ de E.P.) * 4 (Tipo de Problema según la Información ofrecida en el Enunciado: Omiten Información Relevante vs. Soluciones Múltiples vs. Datos Irrelevantes vs. la Solución no requiere Cálculo) con medidas repetidas en el último factor indicó que fue significativo el factor Tipo de Problema según la Información ofrecida en el Enunciado $\left(\mathrm{F}_{(3,126)}=9.741, \mathrm{p}<.01, \eta_{\mathrm{p}}{ }^{2}=.188\right)$, pero no el Grupo (ver Tabla 2$)$. Ninguna de las interacciones alcanzó el nivel de significación.

Tabla 2. Medias y Desviaciones Típicas (entre paréntesis) de las respuestas correctas

\begin{tabular}{lcc}
\hline & $\mathbf{2}^{\mathbf{0}} \mathbf{d e}$ E.P. & $\mathbf{3}^{\mathbf{0}} \mathbf{d e}$ E.P. \\
\hline Omiten Información Relevante & .32 & .50 \\
& $(.57)$ & $(.60)$ \\
Soluciones Múltiples & .23 & .73 \\
& $(.53)$ & $(.83)$ \\
Datos Irrelevantes & .82 & .91 \\
& $(.85)$ & $(.81)$ \\
Solución no requiere Cálculo & .91 & .91 \\
& $(.87)$ & $(.92)$ \\
\hline
\end{tabular}

Puntuación máxima en cada tipo de problema: 2. Nota: se asigna un punto por cada respuesta correcta en cada Tipo de Problema formulado en los ensayos 1 y 2

Adicionalmente, con respecto al factor Tipo de Problema según la Información ofrecida en el Enunciado, el análisis de las comparaciones múltiples, realizado mediante el ajuste de Bonferroni, indicó que las diferencias resultaban significativas entre, por un lado, los problemas que Omiten Información Relevante $(\mathrm{M}=.41, \mathrm{SD}=.58)$ y con Soluciones Múltiples $(\mathrm{M}=$ $.48, \mathrm{SD}=.73)$, que resultaron más complejos y, por otro, los que incluyen Datos Irrelevantes $(\mathrm{M}=.86, \mathrm{SD}=.82)$ y aquéllos cuya Solución no requiere Cálculo $(\mathrm{M}=.91, \mathrm{SD}=.88)$, con una mayor tasa de éxito $(\mathrm{p}<.01$ en todos los casos). 
Análisis de las Respuestas Realistas en función del Tipo de problema según la Información ofrecida en el Enunciado: Respuestas Realistas Correctas e Incorrectas

Los resultados del análisis estadístico mostraron, en primer lugar, que tan sólo el $33.3 \%$ de las respuestas de los niños pudieron considerarse apropiadas, es decir, Respuestas Realistas Correctas. Teniendo en cuenta que la formulación de dichos problemas se realizó de forma que resultaran sencillos, es decir, con una estructura semántica de cambio, ubicando la incógnita en el resultado y empleando cantidades que no alcanzaban la veintena, este bajo porcentaje de aciertos, al igual que el hallado en estudios previos, estaría indicando globalmente el impacto negativo que las creencias inmersas en el contrato didáctico tienen en la resolución de problemas no rutinarios contrapuestos a ellas.

Específicamente, como ya se ha indicado, atendiendo al Tipo de Problema según la Información ofrecida en el Enunciado, los problemas que Omiten Información Relevante y los que tienen Soluciones Múltiples fueron los más complejos. Así, los problemas que Omiten Información Relevante tenían la dificultad de que no era posible realizar cálculos aritméticos $\mathrm{y}$, en el contexto escolar, cuando esto sucede, se considera que los alumnos han fracasado. De forma similar, los problemas con Soluciones Múltiples permitían diversas respuestas numéricas en contra de la tendencia a tratar de hallar una única solución correcta (frente a otras posibles que se consideran incorrectas). Sin embargo, ambos tipos de problema resultaron igualmente complejos para los niños. Estas dificultades se vieron plasmadas, por un lado, en un menor porcentaje de Respuestas Realistas Correctas y, por otro, en la aparición de Respuestas Realistas Incorrectas (ver Tabla 3).

El análisis de las Respuestas Realistas, Correctas e Incorrectas, mostró que, en ambos casos, las dificultades se originaban por el hecho de que no eran capaces de encontrar una respuesta precisa, bien porque no era posible hallar la solución, bien porque era posible más de una solución correcta. Esto es, en los problemas que Omiten Información Relevante, sólo el $20.5 \%$ de las soluciones pudieron ser catalogadas como Respuestas Realistas ya que los niños aludían expresamente a que se desconocía cierta información y, por tanto, la solución no podía calcularse. Por ejemplo, en el problema del "Circo": "sé que Ana le ha dejado 7 euros a María, pero a lo mejor tenía más dinero, así que tiene 7 euros menos de lo que tenía". Paralelamente, vale la pena destacar que algunos niños optaron por realizar cálculos aritméticos cuyo resultado se incluía en la justificación (i.e., 9.1\% de los ensayos), aunque finalmente 
no como solución final al problema, por ejemplo: "20 euros valen las entradas, pero no sé cuánto dinero tiene ahora Ana". Este tipo de respuesta refleja igualmente la necesidad de los niños de realizar cálculos aritméticos e incluirlos en la solución final, aunque dichos cálculos no fuera requeridos por el problema. En el caso de los problemas con Soluciones Múltiples, de forma similar, sólo el $23.9 \%$ de las soluciones totales pudieron ser consideradas Respuestas Realistas Correctas, consistentes en indicar que podría hallarse más de una solución correcta en función del valor numérico que se le asignase al término ambiguo "pocos", como por ejemplo: "9 o más porque en la bolsa de varios sabores hay 1, aunque a lo mejor puede haber más. Más de 1 podría ser, pero ninguno no".

Tabla 3. Porcentaje de ensayos de los tipos de respuesta en cada Tipo de Problema según la Información ofrecida en el Enunciado

\begin{tabular}{lcccc}
\hline & $\begin{array}{c}\text { Omiten } \\
\text { Información } \\
\text { Relevante }\end{array}$ & $\begin{array}{c}\text { Soluciones } \\
\text { Múltiples }\end{array}$ & $\begin{array}{c}\text { Datos } \\
\text { Irrelevantes }\end{array}$ & $\begin{array}{c}\text { La Solución } \\
\text { no requiere } \\
\text { Cálculo }\end{array}$ \\
\hline $\begin{array}{l}\text { Respuestas Realistas } \\
- \text { Respuestas Realistas } \\
\begin{array}{l}\text { Correctas } \\
- \text { Respuestas Realistas }\end{array}\end{array}$ & 20.5 & 23.9 & 43.2 & 45.5 \\
$\begin{array}{l}\text { Incorrectas } \\
\text { Respuestas } \\
\text { No Realistas }\end{array}$ & 5.6 & 14.8 & --- & --- \\
$\begin{array}{l}\text { - Respuestas } \\
\text { Esperadas } \\
\text { - Otras Respuestas }\end{array}$ & 36.4 & 46.6 & 46.6 & 43.2 \\
$\begin{array}{l}\text { Incorrectas } \\
- \text { Ausencia de Res- }\end{array}$ & 36.4 & 13.6 & 10.2 & 10.2 \\
puesta & 1.1 & 1.1 & -- & 1.1 \\
\hline
\end{tabular}

El análisis de las Respuestas Realistas Incorrectas arroja luz sobre los motivos por los cuales los niños encontraron las mismas dificultades en ambos tipos de problemas (ver también Tabla 3). De hecho, estas respuestas sólo aparecen en estos dos tipos de problemas. Así, en los problemas que Omiten Información Relevante, el 5.6\% de las soluciones incluían cierto realismo, pero el error provenía finalmente de que asignaban un valor numérico al dato omitido, lo que les permitía operar y hallar una solución precisa al problema, por ejemplo: "no sé cuánto dinero tenía Ana antes. Si no lo pone, serán 7 euros, así que no le quedaría nada de dinero, 0 euros". 
En el caso de los problemas con Soluciones Múltiples, el intento por parte de los niños de buscar una única solución al problema se tradujo en cuatro tipos de Respuestas Realistas Incorrectas (i.e, $14.8 \%$ de los ensayos). La primera de ellas se produjo porque se omitía el término ambiguo haciendo referencia, por ejemplo, a que "antes no tenía ningún chicle de menta, si luego compra 8 , tiene 8 chicles de menta" (4.5\% de los ensayos). La segunda se originó porque se consideraba inicialmente la información ambigua que aparecía en el problema, pero finalmente no se incorporaba en los cálculos (3.5\% de los ensayos). Un ejemplo de ello, sería la respuesta de uno de los niños en la que se menciona explícitamente que el número de chicles de menta de la primera bolsa no estaba claramente determinado y finalmente lo omitía (p.e., "no lo podemos saber porque no han puesto cuántos pocos había en la bolsa. Si tenía pocos y luego tiene 8, pues tiene 8 chicles de menta en total"). En otros casos, se llegaron a realizar incluso elaboraciones de la historia descrita en el problema para dotar de sentido sus respuestas (p.e., "los pocos chicles que tenía en la bolsa de varios sabores se los come y ya no cuentan, por eso pide los 8 chicles de menta". Este tipo de elaboraciones personales también habían sido documentadas en estudios previos. Por ejemplo, algunos niños habían argumentado en el problema clásico del "Capitán" que éste había comprado una oveja o una cabra en cada cumpleaños y, por eso, era posible conocer la edad que tenía (Freudenthal, 1991; Selter, 1994) e, igualmente, en el problema del “Autobús" los alumnos aludían a que la parte decimal que obtenían en los cálculos de la división (31.33 autobuses) representaba el número de asientos adicionales que eran necesarios (Hatano, 1997).

El tercer tipo de Respuesta Realista Incorrecta consistía en reconvertir o transformar la información ambigua en cantidades numéricas precisas que les permitían operar $(4.5 \%$ de los ensayos). Continuando con el problema de los "Chicles", algunos alumnos interpretaban que: (a) sólo podía haber un chicle de cada sabor, a pesar de que en el texto aparecía explícitamente el término "pocos chicles de menta", de modo que su respuesta final era 9 chicles de menta porque "si antes tenía chicles de varios sabores, en esa bolsa sólo podía tener uno de menta, así que lo sumamos a los 8 que compra" o (b) 16 chicles de menta debido a que "si compra otros 8, es que antes tenía 8 de menta en la bolsa de varios sabores". Este último ejemplo resulta llamativo, puesto que se produjo una compleja transformación de la expresión "compra también 8 chicles de menta" por “compra otros 8 " y fue empleada para justificar la cantidad desconocida. 
Por último, el cuarto tipo de Respuesta Realista Incorrecta se originó porque algunos niños trataban sin éxito de cuantificar aritméticamente la información ambigua $(2.3 \%$ de los ensayos), como ilustra la siguiente respuesta: "podemos sumar 14 más 8 para saber el número de chiches, pero no son todos de menta. Hay 8 que sí, pero en la bolsa que tenía antes ya no lo sé. Entonces, no sé resolver el problema. Yo diría que tengo que hacer otra cuenta para averiguar los pocos que tenía antes, pero no sé cuál". En otras palabras, en esta respuesta se alude explícitamente a que se desconoce la cantidad exacta de chicles de varios sabores y, precisamente, no ser capaz de poder hallarla aritméticamente le condujo al error.

En resumen, a pesar de que estas dos situaciones contradecían creencias escolares diferentes, en ambos tipos de problemas los errores de los niños estuvieron mediatizados por la necesidad de ofrecer una respuesta que fuera numérica y precisa, como pone de manifiesto que en los problemas que Omiten Información Relevante concluyeran que la respuesta era cero y, en los de Soluciones Múltiples, solamente una de las posibles soluciones o una solución idiosincrásica que implicaba igualmente un número preciso.

En segundo lugar, los resultados del análisis estadístico mostraron que los problemas cuya Solución no requiere Cálculo y los que incluían Datos Irrelevantes resultaron más sencillos para los niños (ver Tabla 3). En el primer caso, los niños tenían que identificar que la solución estaba incluida en el enunciado y, por tanto, no era necesario operar. En el segundo, no obstante, la dificultad derivaba de identificar que uno de los datos del problema irrelevante en relación a la pregunta formulada.

En ambos problemas, las Respuestas Realistas Correctas muestran como las justificaciones de los niños estuvieron centradas únicamente en descartar la información del texto que les parecía no significativa. Una vez hecho esto, no resultaba más complejo el hecho de que fuera posible o no realizar cálculos aritméticos. Por ejemplo, en los problemas con Datos Irrelevantes, el $43.2 \%$ de las soluciones fueron Respuestas Realistas Correctas porque los alumnos argumentaban, por ejemplo, en el problema de las "Pinturas" que "sólo importan las 12 que tiene ahora Laura y las 9 que le han regalado, pero los 3 bolígrafos no son pinturas". Igualmente, en los problemas cuya Solución no requiere Cálculo, el $45.5 \%$ de las soluciones se consideraron correctas. Por ejemplo, en el problema de la "Granja”, se basaban en justificaciones tales como: "son las mismas ovejas porque lo que ha comprado son cabras, no ovejas, y las cabras no son ovejas, así que sigue teniendo las mismas que tenía”. Algunos de 
ellos, incluso, mencionaban argumentos adicionales para justificar la no inclusión de uno de los datos del problema aduciendo que "no se pueden incluir los 3 bolígrafos porque no se pueden mezclar las cosas: los árboles, las manzanas...". Por tanto, la creencia consistente en que "para obtener la solución a un problema es necesario operar" no afectó de forma diferencial a las respuestas correctas, aunque también es cierto que esta creencia provocó que algunos niños incluyesen los datos irrelevantes para completar su respuesta final (i.e., "son 21 pinturas y 3 bolígrafos" o "son 25 ovejas y cabras, pero sigue teniendo 17 ovejas"). No obstante, la tendencia a incluir los datos relevantes y no relevantes se aprecia claramente en el análisis de las Respuestas No Realistas.

En ninguno de estos dos tipos de problemas los niños ofrecieron Respuestas Realistas Incorrectas. Si tenemos en cuenta de que este tipo de soluciones reflejaba los comentarios realistas de los niños acompañados de respuestas finales incorrectas, acordes a sus creencias, esta ausencia estaría apoyando el hecho de que estas situaciones eran más sencillas de interpretar para los niños.

Análisis de las Respuestas No Realistas en función del Tipo de problema según la Información ofrecida en el enunciado: Respuestas Esperadas y Otras Respuestas Incorrectas

En cuanto a las Respuestas Esperadas, no se han encontrado grandes diferencias entre los cuatro tipos de problema, como se puede apreciar en la Tabla 3. De hecho, el $42.3 \%$ de las respuestas que ofrecieron los niños fueron codificadas en esta categoría, ya que se produjeron como consecuencia de adicionar todas las cantidades que figuraban en el enunciado. En efecto, numerosos autores habían señalado que los errores que los niños cometen en la resolución de problemas no rutinarios se debían a que seleccionaban todas las cantidades y aplicaban una operación aritmética atendiendo a palabras clave (ver p.e., Carpenter et al., 1983; Fuson, 1992; Hegarty et al., 1995; Orrantia, 2003; Orrantia et al., 2005; Verschaffel y de Corte, 1997). Estos mismos resultados se han visto corroborados en este estudio, puesto que la mayoría de las respuestas de los niños en los cuatro tipos de problema se basaron en la adición y la justificación de dicha operación por medio de palabras clave (p.e., en el problema de los "Chicles", "hay que sumar los 14 chicles que tenía con los 8 que ha comprado porque es de comprar y eso es sumar, no va a ser restar”). 
La presencia de las Otras Respuestas Incorrectas tuvo una mayor incidencia en los tipos de problema que eran más complejos para los niños, esto es, los que Omiten Información Relevante y con Soluciones Múltiples. En concreto, en los primeros, el 36.4\% de las respuestas ofrecidas se obtuvieron mediante la aplicación de procedimientos algorítmicos ajenos a la adición. Así, la mayoría de estas respuestas (i.e., 25\% de los ensayos) estuvieron basadas en la sustracción, por ejemplo, en el problema del "Circo" respondían: "es de quitar el dinero que le deja Ana a María porque si fuera de sumar, tendría más dinero. Igualmente, otras respuestas (i.e., 5.7\%) se basaron en la aplicación de la multiplicación, demostrando una gran incomprensión de dicha operación (i.e., "María tiene 13 euros y su amiga le deja 7 euros y eso es multiplicar"). En los problemas con Soluciones Múltiples, el 13.6\% de los ensayos fueron igualmente Otras Respuestas Incorrectas. En este caso, la operación aritmética mayormente elegida fue la multiplicación (i.e., 6.8\% de los ensayos), indicando que su elección se basaba precisamente en la incomprensión de dicha operación (i.e., “yo sólo entiendo la primera línea y lo de que los preferidos son los de menta, así que multiplico 14 por 8 porque tiene de varios sabores") y, en menor medida, la resta (3.4\%) atendiendo a palabras clave (p.e. "hay que restar porque le han puesto pocos de menta").

Análisis de las Respuestas Realistas en función del Grupo: Respuestas Realistas Correctas e Incorrectas

Con relación al Grupo, el análisis de datos indicó que las diferencias entre $2^{\circ} \quad(\mathrm{M}=$ $.57, \mathrm{SD}=.71)$ y $3^{\circ}$ de E.P. $(\mathrm{M}=.76, \mathrm{SD}=.79)$ no fueron significativas. De hecho, el porcentaje de Respuestas Realistas Correctas e Incorrectas fue similar (ver Tabla 4). Estos datos estarían confirmando la dificultad que supone para los niños enfrentarse a la resolución de problemas no rutinarios, a pesar de que tener un mayor conocimiento sobre las cuatro operaciones aritméticas básicas y una mayor experiencia en la resolución de distintos problemas rutinarios.

Análisis de las Respuestas No-Realistas en función del Grupo: Respuestas Esperadas y Otras Respuestas Incorrectas

Analizando los tipos de errores que cometían los alumnos, en especial las Respuestas Esperadas y las Otras Respuestas Incorrectas, se observaron diferencias entre ambos grupos (ver también Tabla 4). En concreto, el $54.5 \%$ de las respuestas totales de los alumnos de $2^{\circ}$ de 
E.P. fueron clasificadas como Respuestas Esperadas frente al $31.8 \%$ en el caso de los de $3^{\circ}$ de E.P. Como ya se ha señalado en la introducción, diversos autores habían señalado que los niños, en ocasiones, no se guían por la operación que sugiere la estructura del problema, sino que tienden a operar empleando la última operación aritmética aprendida (p.e., Davis, 1989; De Corte y Verschaffel, 1985; Freudenthal, 1991; Greer, 1993; Kilpatrick, 1987; Nesher, 1980; Reusser, 1988; Reusser y Stebler, 1997; Säljö, 1991; Schoenfeld, 1991; Silver et al., 1993; Treffers y De Moor, 1990; Verschaffel y De Corte, 1997). Esto explicaría que, en $3^{\circ} d e$ E.P., el $26.7 \%$ de los errores se produjeran por la aplicación de operaciones aritméticas ajenas a la adición frente al $8.5 \%$ en $2^{\circ}$ de E.P. Es decir, los niños de $2^{\circ}$ aplicaron la resta tan sólo en el $6.8 \%$ de las ocasiones y la combienación de las operaciones de sumar y restar en el 1.7\% de las situaciones. Sin embargo, un mayor porcentaje de las respuestas de los niños de $3^{o}$ se basaron en la aplicación de la resta (9.1\%), la multiplicación (8.5\%), la combinación de dos o más operaciones aritméticas (8\%) e, incluso, aunque en menor medida, a la división (1.1\%), acompañadas de argumentos que mostraban la incomprensión de dichas operaciones o simplemente leyendo el enunciado como justificación.

Tabla 4. Porcentaje de ensayos de los tipos de respuesta en función del Grupo

\begin{tabular}{lcc}
\hline & $\mathbf{2}^{\mathbf{0}}$ de E.P. & $\mathbf{3}^{\mathbf{0}}$ de E.P. \\
\hline Respuestas Realistas & & \\
- Respuestas Realistas Correctas & 28.4 & 38.1 \\
- Respuestas Realistas Incorrectas & 8.0 & 2.2 \\
\hline Respuestas No Realistas & & \\
- Respuestas Esperadas & 54.5 & 31.8 \\
- Otras Respuestas Incorrectas & 8.5 & 26.7 \\
- Ausencia de Respuesta & .6 & 1.2 \\
\hline
\end{tabular}

\section{Discusión y Conclusiones}

La primera conclusión que se extrae de los resultados del análisis de las Respuestas Realistas Correctas e Incorrectas, en función del Tipo de Información ofrecida en el Enunciado, es que se confirma que los alumnos tienden a resolver los problemas aritméticos en función de las normas que imperan en el aula. Precisamente, son esas normas las que, desde nuestro punto de vista, dificultan que los niños resuelvan los problemas aplicando el conocimiento que poseen sobre el mundo real. En el presente trabajo, principalmente tres resultados sustentan esta afirmación. 
En primer lugar, la dificultad de los problemas se ha visto afectada por las creencias que éstos contravenían. En otras palabras, las creencias incorrectas que rigen el contrato didáctico en el aula parecen ser las responsables de la mayor o menor dificultad que los alumnos han encontrado a la hora de resolver los problemas. Así, los resultados de este estudio estarían aportando evidencia empírica favorable sobre que algunas creencias están más asentadas en el pensamiento infantil que otras. Para los niños, las situaciones más difíciles de resolver serían aquéllas en las que no se puede hallar una solución precisa, bien porque no exista una solución, bien debido a que resulta inverosímil la posibilidad de que haya más de una solución correcta. Igualmente, aunque fueron situaciones más sencillas, los niños también encuentran dificultades para detectar que la solución al problema está incluida en el resultado o para descartar en los cálculos cierta información irrelevante.

En segundo lugar, algunas de las respuestas correctas muestran claramente que la dificultad no se debe a la incapacidad de los alumnos para realizar consideraciones realistas, sino a la tendencia a seguir fielmente el modo de proceder habitual en el aula. Es decir, en palabras de Caldwell (1995), los niños consideran que deben resolver de forma diferente los problemas escolares y los que acontecen en el mundo real. En este trabajo, algunas respuestas de los niños muestran claramente sus dudas e incertidumbre ante el hecho de que estos problemas no se ajusten a los típicamente presentados en clase de matemáticas (p.e., en el problema de los "Chicles" "no lo entiendo. No entiendo qué es lo que tiene de varios sabores y no sé cuántos son de menta. Bueno, en una bolsa de varios sabores suele venir uno, si hay uno son 9 de menta, si hay dos son 10") e, incluso, plantean dudas sobre la "honradez" de los problemas (p.e., "es un problema con truco, tenía cebo para picar").

Por último, muchos comentarios realistas a los problemas generan soluciones incorrectas. En estos casos, tampoco se pueden atribuir los errores al hecho de que no fueran capaces de considerar los aspectos realistas de los problemas, sino a que los niños finalmente actúan conforme a lo que creen que se espera de ellos. Este resultado sintoniza con la defensa de que es importante que los estudiantes se sientan libres de enfrentarse a los problemas de forma realista.

Una segunda conclusión que puede extraerse de este estudio es la similar ejecución de los alumnos de $2^{\circ}$ y $3^{\circ}$ de E.P. Es decir, a pesar de que los niños de $3^{\circ}$ de E.P. tienen una mayor experiencia en resolución de problemas y conocen las cuatro operaciones aritméticas 
básicas, su rendimiento no ha sido significativamente superior al de los niños de $2^{\circ}$. Es más, resulta llamativo que, para resolver problemas con una estructura aparentemente aditiva, muchos de los errores de los niños de $3^{\circ}$ se hayan producido por la aplicación de la resta, la multiplicación, la división o la combinación de dos operaciones aritméticas. Este dato confirma igualmente la tendencia de algunos niños a operar en función de palabras clave, cuando se decantaron por la adición, o a seleccionar la última operación aritmética estudiada, demostrando con ello la incompresión sobre la función de dichas operaciones a la hora de resolver problemas.

En definitiva, la conclusión final que se desprende de este trabajo es que los niños resuelven los problemas de forma mecánica, no preguntándose realmente qué se les está demandando. Tal y como se establece en el Real Decreto 1513/2006, de 7 de diciembre, por el que se establecen las enseñanzas mínimas de la Educación Primaria, una de las competencias básicas en el área de matemáticas consiste en "la habilidad para utilizar y relacionar los números, sus operaciones básicas, los símbolos y las formas de expresión y razonamiento matemático para resolver problemas relacionados con la vida cotidiana" y uno de los criterios de evaluación debería ser "la resolución con éxito de problemas sencillos relacionados con objetos, hechos y situaciones de la vida cotidiana utilizando los algoritmos básicos”. Tal y como se ha mostrado en este estudio, los problemas rutinarios no parecen estar contribuyendo a que se alcancen dichos requisitos educativos. Partiendo de los resultados anteriormente expuestos, sería deseable realizar cambios en los problemas que típicamente se presentan en el aula, de forma que se aumente el realismo y autenticidad de los mismos. Los problemas no rutinarios, en los que los alumnos tengan que juzgar, tal y como sucede en la vida real, si es posible que un problema no tenga una solución aritmética, que varias alternativas sean válidas, que cierta información sea superflua o, por último, que un problema pueda presentarse previamente resuelto, serían una propuesta a considerar para ayudar a que los alumnos alcancen esta competencia. De esta manera, estos problemas estarían ayudando a que los niños no asumiesen determinadas creencias incorrectas que subyacen al contrato didáctico. 
El impacto negativo del contrato didáctico en la resolución realista de problemas. Un estudio con alumnos de $2^{\circ}$ y $3^{\circ}$ de Educación Primaria

\section{Referencias}

Baruk, S. (1985). L'âge du capitaine. De l'erreur en mathematiques. París: Seuil.

Becker, J. y Selter, C. (1996). Elementary school practices. En A. Bishop, K. Clements, C. Keitel, J. Kilpatrick y C. Laborde. (Eds.). International Handbook of Mathematics Education (pp. 511-564). Dordrecht: Kluwer Academic Publishers.

Blum, W. y Niss, M. (1991). Applied mathematical problem solving, modelling, applications, and links to other subjects-state, trends and issues in mathematics instruction. Educational Studies in Mathematics, 22, 37-68.

Boaler, J. (1993). The role of contexts in the mathematics classroom: Do they make mathematics more "real"? For the Learning of Mathematics, 13(2), 12-17.

Brousseau, G. (1984). The IREM's role in helping elementary-school teachers. En R. Morris (Ed.), Studies in mathematics education. The mathematical education of primaryschool teachers, vol. 3 (pp. 235-251). París: UNESCO.

Burkhardt, H. (1994). Mathematical applications in school curriculum. En T. Husén y T.N. Postlethwaite (Eds.), The international encyclopaedia of education 2nd ed. (pp. 36213624). Oxford, New York: Pergamon Press.

Cai, J. y Silver, E. A. (1995). Solution processes and interpretations of solutions in solving a division with remainder story problems: Do Chinese and U.S. students have similar difficulties? Journal for Research in Mathematics Education, 26, 491-497.

Caldwell, L. (1995). Contextual considerations in the solution of children's multiplication and division word problems. Tesis doctoral no publicada, Belfast, Northern Ireland: Queen's University.

Carpenter, T. P., Hiebert, J. y Moser, J. M. (1983). The effect of instruction on children's solutions of addition and subtraction word problems. Educational Studies in Mathematics, 14, 55-72.

Carpenter, T. P., Lindquist, M. M., Matthews, W. y Silver, E. A. (1983). Results of the third NAEP mathematics assessment: Secondary school. Mathematics Teacher, 76, 652659.

Davis, R. B. (1989). The culture of mathematics and the culture of schools. Journal of Mathematical Behavior, 8, 143-160. 
De Corte, E. y Verschaffel, L. (1985). Beginning first graders' initial representation of arithmetic word problems. Journal of Mathematical Behaviour, 4, 3-21.

Ellerton, N. y Clarkson, P. (1996). Language factors in mathematics teaching and learning. En A. Bishop, K. Clements, C. Keitel, J. Kilpatrick y C. Laborde (Eds.), International Handbook of mathematical education (pp. 987-1034). Dordrecht: Kluwer Academic Publishers.

Freudenthal, H. (1991). Revisiting mathematics education. Dordrecht: Kluwer.

Fuson, K. C. (1992). Research on whole number addition and subtraction. En D. Grouws (Ed.), Handbook of research on mathematics teaching and learning (pp. 243-275). Nueva York: MacMillan.

Gil, N., Guerrero, E., y Blanco, L. (2006). El dominio afectivo en el aprendizaje de las matemáticas. Electronic Journal of Research in Educational Psychology, 4(1), 47-72.

Greer, B. (1993). The mathematical modeling perspective on wor(1)d problems. Journal of Mathematical Behaviour, 12(3), 239-250.

Hatano, G. (1997). Cost and benefit of modeling activity. Commentary. Learning \& Instruction, 7(4), 383-387.

Hegarty, M., Mayer, R. E. y Monk, C. (1995). Comprehension of arithmetic word problems: A comparison of successful and unsuccessful problem solvers. Journal of Educational Psychology, 87, 18-32.

Hidalgo, M. C. (1997). L'activation des connaissances à propos du monde réel dans la résolution de problèmes verbaux en arithmétique. Tesis doctoral no publicada, Québec, Canada: Université Laval.

Jiménez, L. (2008). La activación del conocimiento real en la resolución de problemas: un estudio evolutivo sobre los problemas no-rutinarios de adición. Tesis doctoral, Madrid: Universidad Complutense.

Kilpatrick, J. (1987). Problem formulating: Where do good problems come from? In A.H. Schoenfeld (Ed.), Cognitive science and mathematics education (pp. 123-147). Hillsdale, NJ: Erlbaum. 
Lago, M. O., Rodríguez, P., Enesco, I., Jiménez, L. y Dopico, C. (2008). "Me sobran cuatro y no sé qué hacer con ellos". Un estudio sobre los problemas de división con resto en alumnos de $1^{\circ}$ de ESO. Anales de Psicología, 24(2), 201-212.

Li, Y. y Silver, E. A. (2000). Can younger students succeed where older students fail? An examination of third graders' solutions of a division-with-remainder (DWR) problem. Journal of Mathematical Behavior, 19, 233-246.

Nelissen, J. M. C. (1987). Kinderen leren wiskunde. Een studie over constructie en reflectie in het basisonderwijs [Children learn mathematics. A study concerning construction and reflection in elementary school education]. Gorinchem: De Ruiter.

Nesher, P. (1980). The Stereotyped Nature of School word problems. For the Learning of Mathematics, 1(1), 41-48.

Niss, M. (1996). Goals of mathematics teaching. En A. Bishop, K. Clements, C. Keitel, J. Kilpatrick y C. Laborde (Eds.), International Handbook of mathematical education (pp. 11-48). Dordrecht: Kluwer Academic Publishers.

Orrantia, J. (2003). El rol del conocimiento conceptual en la resolución de problemas aritméticos con estructura aditiva. Infancia y Aprendizaje, 26(4), 451-468.

Orrantia, J., González, L. B. y Vicente, S. (2005). Estudios del conocimiento numérico: Aprendizaje y enseñanza. Un análisis de los problemas aritméticos en los libros de texto de Educación Primaria. Infancia y Aprendizaje, 28(4), 429-451.

Palm, T. (2006). Word problems as a simulations of real-world situation: A proposed framework. For the Learning of Mathematics, 26(1), 42-47.

Palm, T. (2008). Impact of authenticity on sense-making in word problem solving. Educational Studies in Mathematics, 67(1), 37-58.

Palm, T. (2009). Theory of authentic task situations. En L. Verschaffel, B. Greer, W.V. Dooren y S. Mukhopadhyay (Eds.), Words and Worlds, Modelling verbal descriptions of situations (pp. 3-19). Rotterdam, The Netherlands: Sense Publishers.

Real Decreto 1513/2006, de 7 de diciembre, por el que se establecen las enseñanzas mínimas de la Educación Primaria (BOE, n 293, viernes 8 de diciembre de 2006).

Reusser, K. (1988). Problem solving beyond the logic of things: Contextual effects on understanding and solving word problems. Instructional Science, 17, 309-338. 
Reusser, K. y Stebler, R. (1997). Every word problem has a solution: The suspension of reality and sense-making in the culture of school mathematics. Learning \& Instruction, 7, 309-328.

Rodríguez, P., Lago, M. O., Hernández, M. L., Jiménez, L., Guerrero, S. y Caballero, S. (2009). How do secondary students approach different types of division with remainder situations? Some evidence from Spain. European Journal of Educational Psychology, 24(4), 529-543.

Säljö, R. y Wyndhamn, J. (1987). The formal setting as context for cognitive activities. An empirical study of arithmetic operations under conflicting premises for communication. European Journal of Psychology of Education, 2(3), 233-245.

Schoenfeld, A. H. (1991). On mathematics as sense-making: An informal attack on the unfortunate divorce of formal and informal mathematics. En J.F. Voss, D.N. Perkins y J.W. Segal (Eds.), Informal reasoning and education (pp. 311-343). Hillsdale, NJ: Lawrence Erlbaum Associates.

Selter, C. (1994). How old is the captain? Strategies, 5(1), 34-37.

Silver, E. A. (1986). Using conceptual and procedural knowledge: A focus on relationships. En J. Hiebert (Ed.), Conceptual and procedural knowledge:The case of mathematics (pp. 181-197). Hillsdale, NJ: Lawrence Erlbaum.

Silver, E. A., Mukhopadhyay, S. y Gabriele, A. J. (1992). Referential mappings and the solution of division story problems involving remainders. Focus on Learning Problems in Mathematics, 14, 29-39.

Silver, E. A., Shapiro, L. J. y Deutsch, A. (1993). Sense making and the solution of division problem involving remainders: An examination of middle school students' solution processes and their interpretations of solutions. Journal for Research in Mathematics Education, 24, 117-135.

Treffers, A. y De Moor, E. (1990). Proeve van een nationaal programma voor het rekenwiskundeonderwijs op de basisschool. Dee12: Basisvaardigheden en cijferen [Specimen of a national program for primary mathematics teaching. Part 2: Basic mental skills and written algorithms]. Tilburg, The Netherlands: Zwijsen.

Verschaffel, L. Greer, B. y De Corte, E. (2000). Making sense of word problems. Lisse: Swets \& Zeitlinger. 
Verschaffel, L. y De Corte, E. (1997). Word problems: A vehicle for promoting authentic mathematical understanding and problem solving in the primary school? En T. Nunes y P. Bryant (Eds.), Learning and teaching mathematics: An international perspective (pp. 69-97). Hove, East Sussex: Psychology Press Ltd.

Verschaffel, L., De Corte, E. y Lasure, S. (1994). Realistic considerations in mathematical modelling of school arithmetic word problems. Learning \& Instruction, 4, 273-294.

Verschaffel, L., De Corte, E. y Lasure, S. (1999). Children's conceptions about the role of real-world knowledge in mathematical modelling of school word problems. En W. Schnotz, S. Vosniadou y M. Carretero (Eds.), New perspectives on conceptual change (pp. 175-189). Oxford: Elsevier.

Verschaffel, L., Greer, B. Van Dooren, W. V. y Mukhopadhyay, S. (2009). Words and Worlds. Modelling verbal descriptions of situations. Rotterdam, The Netherlands: Sense Publisher.

Vicente, S., Van Dooren, W. V. y Verschaffel, L. (2008). Utilizar las matemáticas para resolver problemas reales. Cultura \& Educación, 20(4), 391-406.

Villegas, J. L., Castro, E. y Gutierrez, J. (2009). Representaciones en resolución de problemas: Un estudio de caso con problemas de optimización. Electronic Journal o Research in Educational Psychology, 7(1), 279-308.

Wyndhamn, J. y Säljö, R. (1997). Word problems and mathematical reasoning: A study of children's mastery of reference and meaning in textual realities. Learning \& Instruction, 7, 361-382.

Yoshida, H., Verschaffel, L. y De Corte, E. (1997). Realistic considerations in solving problematic word problems: Do Japanese and Belgian children have the same difficulties? Learning \& Instruction, 7, 329-338. 
Jiménez, L. et al.

[Pagina en blanco por razones de paginación] 